\title{
Análise bibliométrica de mobilidade urbana
}

\section{Victor Hugo-Pereira}

Programa de Pós-Graduação em Planejamento e Governança Pública, Universidade Tecnológica Federal do Paraná, Brasil. http://lattes.cnpq.br/9887685785954461

Recibido: 29 de julio de 2019. Aceptado: 31 de octubre de 2019.

\begin{abstract}
Resumo
O estudo de Mobilidade Urbana subsidia ações públicas e privadas que proporcionem deslocamentos sustentáveis para a plenitude da cidadania. O presente trabalho visa realizar um estudo bibliométrico que permita selecionar artigos científicos de grande relevância, identificando o estado da arte sobre o tema, por meio de busca de artigos científicos publicados em periódicos indexados às principais bases de pesquisa encontrados no sítio eletrônico Periódicos da Coordenação de Aperfeiçoamento de Pessoal de Nível Superior (CAPES) utilizando a palavra-chave "mobilidade urbana" no período de 2012 a 2018 em um processo estruturado de seleção chamado Knowledge Development Process Constructivist - Proknow C. De um total de 268 trabalhos, foram selecionados 31 artigos de maior relevância no tema Mobilidade Urbana, cujos autores e periódicos de maior destaque foram classificados mediante análise bibliométrica, concluindo que a pesquisa relacionada à Mobilidade Urbana é extensa, abordando aspectos socioeconômicos, ambientais, sanitários, produtivos e de qualidade de vida da população, contudo, se concentra em um restrito grupo de pesquisadores e periódicos.
\end{abstract}

Palavras-chave: Mobilidade Urbana. Transporte. Análise Bibliométrica.

\section{Bibliometric analysis of Urban Mobility}

\begin{abstract}
The Urban Mobility study subsidizes public and private actions that provide sustainable displacement for the fullness of citizenship. The present work aims to conduct a bibliometric study that allows the selection of scientific articles of great relevance, identifying the state of the art on the subject, by searching scientific articles published in journals indexed to the main research bases found in the Periódicos da Coordenação de Aperfeiçoamento de Pessoal de Nível Superior (CAPES) website using keyword "urban mobility" from 2012 to 2018 in a structured selection process called
\end{abstract}


Knowledge Development Process Constructivist - Proknow C. From a total of 268 papers, 31 most relevant articles were selected on the theme Urban Mobility, whose authors and highlighted journals were classified by bibliometric analysis, concluding that the research related to Urban Mobility is extensive, addressing socioeconomic, environmental, health, productive and quality of life aspects of the population, however, it focuses on a restricted group of researchers and journals.

Keywords: Urban Mobility. Transport. Bibliometric Analysis.

Palabras clave: Movilidad urbana. Transporte. Análisis bibliométrico.

\section{Introdução}

A crescente migração da população rural para as áreas urbanas é um fenômeno contemporâneo. No Brasil, o Instituto Brasileiro de Geografia e Estatística - IBGE aponta que nos anos 1960 o país ainda era agrícola, com uma taxa de urbanização de apenas $44,7 \%$, sendo que nos anos 1990 essa taxa saltou para $75,59 \%$ chegando a $84,36 \%$ em 2010 (IBGE, 2010).

Em face ao crescimento expressivo das cidades brasileiras verificado nas últimas décadas, o conceito de mobilidade tem ganhado importância, geralmente acompanhado do paradigma do desenvolvimento sustentável que surgiu no final da década de 1980. mobilidade vêm a substituir o termo "transporte", haja vista ser mais abrangente, passando a ideia de deslocamento de pessoas e considerando também os meios de locomoção não motorizados (Machado y Lima, 2015; Silva, 2013).

A mobilidade entra em choque com o pensamento dicotômico clássico entre espaços públicos e espaços de circulação, no qual esse último tem caráter essencialmente mecânico atribuído às redes de transporte, reduzidas à função de circulação. Com o advento da perspectiva do usuário nos problemas de transporte, a mobilidade passou a ser vista como um direito à cidadania (Mello y Portugal, 2017; Silva, 2016).

A mobilidade urbana surge como conceito de deslocamento de pessoas e bens dentro dos espaços das cidades com ênfase no indivíduo em detrimento aos veículos e tornando o direito à cidade acessível a todos (Haddad y Vieira, 2015; Machado y Lima, 2015; Pero y Stefanelli, 2015; Vianna y Young, 2015; Wilheim, 2013).

Diante deste cenário são necessários estudos técnicos e científicos que embasem o desenvolvimento de adequadas políticas públicas de desenvolvimento urbano, por meio de planos diretores e setoriais, em especial, os planos de mobilidade urbana (Gomide y Galindo, 2013; Pero y Stefanelli, 2015; Silva, 2013; Wilheim, 2013).

Em plena era do conhecimento, o acesso aos mais diversos estudos e trabalhos de pesquisa elaborados no Brasil e no mundo, por meio da internet, tornou-se fácil, mas também, um desafio para os pesquisadores. Em relação ao tema mobilidade urbana, por exemplo, foram encontrados no portal de Periódicos da Coordenação de Aperfeiçoamento de Pessoal de Nível Superior - CAPES 268 trabalhos no período de 6 anos de busca. A abundância de material pode vir a prejudicar a qualidade final da pesquisa, requerendo assim um planejamento prévio de seleção de fontes de pesquisa (Marafon et al., 2012).

Neste cenário, o uso da bibliometria como técnica quantitativa e estatística de medição dos índices de produção e disseminação do conhecimento científico (Araújo, 2006) pode facilitar a identificação de estudos e trabalhos apropriados para a proposta de pesquisa. 
Segundo Araújo (2006), o estudo bibliométrico, surgido como forma de avaliação da produção científica no início do século XX, engloba a análise de citação como importante instrumento para verificar a relação entre os documentos citantes com os documentos citados, permitindo a identificação e descrição de uma série de padrões na produção do conhecimento científico.

É possível identificar a elite da pesquisa, com autores mais citados e mais produtivos, o fator de impacto dos autores, procedência geográfica e institucional dos autores mais influentes, a obsolescência da literatura, entre outras importantes análises (Ensslin, Ensslin y Pinto, 2013).

Com o objetivo de enriquecer o estudo bibliométrico, a elaboração de um processo estruturado para a seleção e análise da literatura científica, que torna a pesquisa ainda mais robusta, Ensslin, Ensslin, Pinto (2013) indicam o instrumento Knowledge Development Process Constructivist - Proknow C. O processo Proknow-C é composto por quatro etapas: a seleção de um portfólio de artigos sobre o tema da pesquisa, a análise bibliométrica e sistêmica do portfólio e definição de pergunta de pesquisa e objetivo, respectivamente.

\section{Estudo bibliométrico}

A formação do portfólio de artigos reúne o conjunto de artigos relacionados e alinhados ao tema de pesquisa, por meio da percepção e das delimitações pré-determinadas pelos pesquisadores.

Para atender os propósitos do presente estudo foram utilizadas duas das quatro etapas do Proknow-C: a seleção de um portfólio de artigos sobre o tema da pesquisa e a análise bibliométrica.

A formação do portfólio de artigos reúne o conjunto de artigos relacionados e alinhados ao tema de pesquisa, por meio da percepção e das delimitações pré-determinadas pelos pesquisadores.

A primeira etapa pode ser segmentada em três fases: (1) a seleção dos artigos nas bases de dados que compõem o Banco de Artigos Bruto; (2) a filtragem dos artigos selecionados com base no alinhamento da pesquisa; e (3) o teste de representatividade do Portfólio Bibliográfico (Figura 1).

\section{Seleção de artigos $>$ Filtragem dos artigos $>$ Teste de representatividade}

Figura 1. Fases da Etapa de Seleção do Portfólio Bibliográfico. Fonte: Elaboração própria.

O resultado dessa etapa representa o conjunto de artigos mais relevantes e alinhados com a pesquisa, denominando-se de Portfólio Bibliográfico (PB). Cabe ressaltar que os procedimentos realizados nesta pesquisa ocorreram de outubro de 2018 a fevereiro de 2019.

A segunda etapa realiza-se uma análise dos artigos com o objetivo de quantificar as informações existentes e fornecer as características das publicações. São considerados os aspectos de relevância e fator de impacto dos periódicos, reconhecimento científico dos artigos e autores de maior destaque (Figura 2). 


\begin{tabular}{c|c|c|c|}
$\begin{array}{c}\text { Relevância dos } \\
\text { periódicos }\end{array}$ & $\begin{array}{c}\text { Fator de impacto } \\
\text { dos periódicos }\end{array}$ & $\begin{array}{c}\text { Reconhecimento } \\
\text { científico dos artigos }\end{array}$ & $\begin{array}{c}\text { Autores de maior } \\
\text { destaque }\end{array}$ \\
\hline
\end{tabular}

Figura 2. Fases da Etapa de Análise Bibliométrica. Fonte: Elaboração própria.

\section{Etapa 1: Seleção de um portfólio de artigos sobre o tema da pesquisa}

A definição do eixo de pesquisa se faz necessária para o processo de seleção do banco de artigos bruto, sendo que para este estudo, o eixo definido foi o de mobilidade urbana, permitindo ao pesquisador direcionar a construção do conhecimento necessário ao redor de seu contexto.

As ações seguintes para a formação do Banco de Artigos Brutos são (1) a definição das palavras-chave; (2) a definição da base de dados; (3) a busca pelos artigos nas bases de dados com as palavras-chave; $\mathrm{e}(4)$ a realização de teste de aderência das palavras-chave.

A primeira fase da etapa de seleção dos artigos brutos se deu com a definição das palavras-chave do eixo citado, sendo definida a palavra-chave "mobilidade urbana". A seguir, com o eixo de pesquisa e a palavra-chave definidos, passou-se para a seleção das bases de dados disponibilizadas no Portal de Periódicos da CAPES, alinhadas com a área de conhecimento considerada relevante para a pesquisa, no caso as Ciências Sociais Aplicadas.

O Portal de Periódicos da CAPES é uma biblioteca virtual que reúne e disponibiliza a instituições de ensino e pesquisa no Brasil acesso a produção científica internacional, contando com um acervo de mais de 45 mil títulos com texto completo e 130 bases referenciais.

Foram identificadas e selecionadas as 72 bases de dados relacionadas à área de Ciências Sociais Aplicadas no Portal de Periódicos da CAPES (Brasil. Ministério Da Educação, s/f), a destacar as bases Web of Science, Scopus, Emerald, Proquest, Scielo, Science Directe Informs, as quais indexam uma gama de periódicos científicos alinhados ao tema da pesquisa.

Com as bases definidas, iniciou-se a busca utilizando a palavra-chave definida no eixo de pesquisa, restringindo-se aos campos de títulos dos artigos, palavras-chave e resumos. A esses critérios de restrição, estabeleceu-se um período temporal de 6 anos anteriores à pesquisa (2012 a 2018), de trabalhos publicados apenas em periódicos com revisão por pares (peer review).

As buscas realizadas nas bases de periódicos a partir da utilização da palavra-chave definidas encontrou um total de 268 trabalhos publicados, que passaram a compor o Banco de Artigos Bruto. Para a reunião dos trabalhos e composição dos Bancos de Artigo foi utilizado o software Mendeley como gerenciador bibliográfico.

A próxima fase foi o teste de aderência das palavras-chave, no qual foram selecionados cinco artigos, aleatoriamente, com o objetivo de identificar a necessidade de inclusão de novas palavras-chave. Após a execução dos procedimentos, concluiu-se que o alinhamento entre a palavra-chave e os artigos com o tema de pesquisa, dispensando a inclusão de novas palavras-chave. Com as fases concluídas, iniciou-se a etapa da filtragem dos artigos brutos.

Alguns aspectos foram levados em consideração no processo de filtragem, como (1) a presença de artigos repetidos/redundantes; (2) o alinhamento dos títulos dos artigos com o tema; (3) o reconhecimento científico dos artigos; (4) alinhamento dos resumos com o tema; e (5) a disponibilidade dos artigos na íntegra nas bases. 
Partindo de uma base de 268 artigos foi possível identificar e excluir os artigos redundantes, resultando em 16 artigos excluídos e restando 252 artigos para a análise de alinhamento de títulos.

Após a leitura de todos os títulos, identificou-se 146 artigos com títulos alinhados com o tema da pesquisa, sendo listados em uma planilha para verificação do grau de reconhecimento científico das publicações, por meio de consulta ao Google Acadêmico (Google, s/f), objetivando calcular a quantidade de citações de cada um dos trabalhos.

Realizado o levantamento de citações, foram fixadas como representativas $90 \%$ das citações dos trabalhos, sendo 10\% compõem-se de artigos com menos de quatro citações. Em números absolutos, 47 artigos ( $90 \%$ das citações) passaram a compor o repositório de títulos alinhados e com reconhecimento científico, a ser chamado de repositório K.

Os outros 99 artigos (10\% das citações) foram separados em outro repositório chamado repositório $\mathrm{P}$, que classifica os títulos alinhados com menor reconhecimento científico ou com reconhecimento ainda não confirmado.

Próximo passo é a leitura dos resumos dos 47 artigos do repositório k para verificar o alinhamento do conteúdo com o tema. Destes 47, 27 se enquadraram ao tema e foram classificados como integrantes de um novo repositório A, excluindo-se os trabalhos restantes do repositório $\mathrm{K}$, não enquadrados. Os autores dos artigos do repositório $\mathrm{A}$ passaram a formar o Banco de Autores (BA).

Na sequência, o repositório P passa a ser analisado a fim de identificar outros artigos com potencial para compor o Portfólio Bibliográfico, sendo que dos 99 artigos do repositório, 57 foram publicados entre 2016 e 2018, alguns dos quais sem qualquer reconhecimento científico, mas com potencial de aproveitamento de seu conteúdo por serem recentes.

Destes 57 foram selecionados 2 artigos recentes (2016 a 2018) escritos por autores constantes do Banco de Autores, passando a integrar o novo repositório B.

O passo a seguir é a leitura dos resumos dos artigos com intuito de verificar o alinhamento com o tema, sendo que dos 27 do repositório A e dos 2 do repositório B foram selecionados 28 artigos para compor o novo repositório C, devido ao alinhamento dos resumos com o tema de pesquisa.

Na fase seguinte à filtragem, verificou-se a disponibilidade na íntegra dos artigos, sendo que a não gratuidade destes se tornaria um limitador ao trabalho. Todos os 28 artigos foram encontrados na íntegra por meio do Portal de Periódicos da CAPES.

Passou-se para leitura integral dos artigos, a fim de confirmar o alinhamento com o tema da pesquisa, encontrando-se 27 (novo repositório D) deles alinhados, encerrandose aqui o processo de filtragem dos artigos.

A próxima fase é do teste de representatividade do Portfólio Bibliográfico, com objetivo de analisar as referências bibliográficas citadas nos artigos do repositório D. Levantaram-se todas as referências bibliográficas nos artigos, restringindo-se ao espaço temporal considerado para estudo de 2012 a 2018 e a artigos publicados em periódicos.

Para facilitar a composição das referências dos artigos do Portfólio Bibliográfico, mais uma vez, utilizou-se do gerenciador bibliográfico Mendeley, para que os dados fossem exportados para uma planilha, para posterior filtragem e análise de representatividade das citações. 
Com a planilha montada e organizada por artigos, foi realizada uma nova consulta ao sítio do Google Acadêmico para identificar a quantidade de citações feitas aos artigos das referências do Portfólio Bibliográfico, reorganizando-se a planilha de forma decrescente e estabelecendo-se o grau de representatividade em percentuais de cada artigo em relação ao total de referências.

Neste processo de mapeamento foram selecionados $80 \%$ das citações totais a fim de identificar se os artigos do Portfólio Bibliográfico continham essas referências, aspecto que será evidenciado na análise bibliométrica deste artigo.

Dos artigos citados nas referências foram identificados 3 autores de destaque com um número elevado de citações e com alinhamento com o tema, os quais foram incorporados ao Portfólio Bibliográfico, totalizando 31 artigos (Figura 3) enquadrados ao tema, detalhados no Quadro 1.

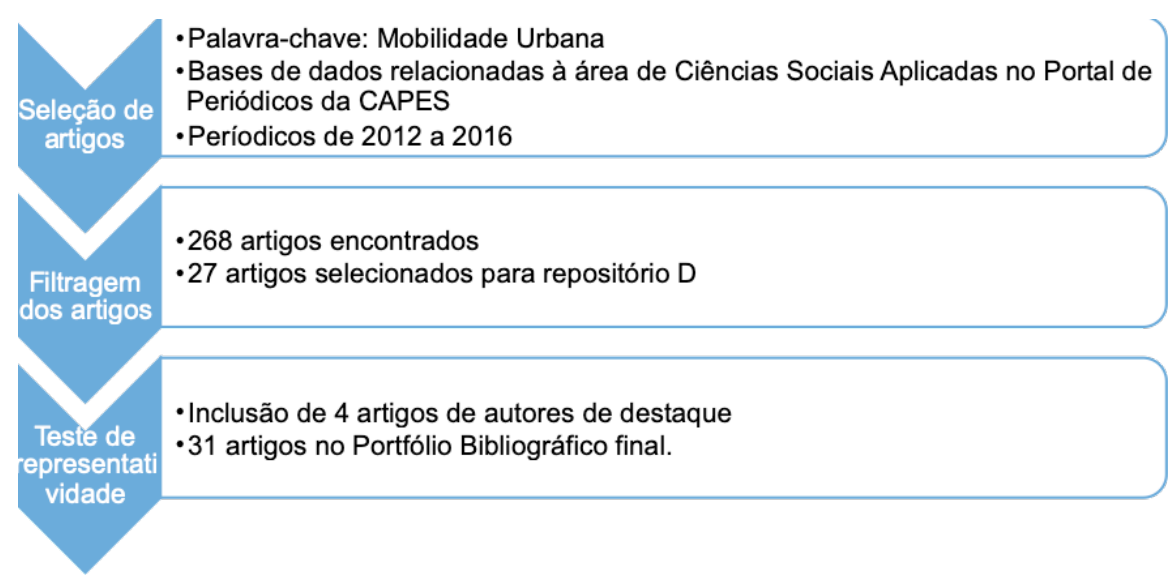

Figura 3. Execução da Seleção do Portfólio Bibliográfico. Fonte: Elaboração própria.

Em busca realizada em janeiro de 2019 foram localizados 268 trabalhos publicados, que passaram por processo de filtragem de artigos, do qual restaram, após avaliação e leitura, apenas 31 artigos relevantes ao assunto e que passaram a compor o Portfólio Bibliográfico (PB) da pesquisa (Quadro 1).

Quadro 1. Portfólio Bibliográfico de Mobilidade Urbana. Fonte: Elaboração própria.

\begin{tabular}{|l|c|c|}
\hline Artigos e autores & Ano & Citações \\
\hline $\begin{array}{l}\text { The key to sustainable urban development in UK cities? The influence of } \\
\text { density on social sustainability } \\
\text { (Dempsey, Brown y Bramley, 2012) }\end{array}$ & 2012 & 222 \\
\hline $\begin{array}{l}\text { Linking urban transport and land use in developing countries } \\
\text { (Cervero, 2013) }\end{array}$ & 2013 & 221 \\
\hline $\begin{array}{l}\text { Urban sustainable transportation indicators for global comparison } \\
\text { (Haghshenas y Vaziri, 2012) }\end{array}$ & 2012 & 180 \\
\hline $\begin{array}{l}\text { O plano de mobilidade urbana e o futuro das cidades } \\
\text { (Rubim y Leitão, 2013) }\end{array}$ & 2013 & 52 \\
\hline $\begin{array}{l}\text { Impactos ambientais da mobilidade urbana: cinco categorias de medidas } \\
\text { mitigadoras } \\
\text { (Barczak y Duarte, 2012) }\end{array}$ & 2012 & 50 \\
\hline $\begin{array}{l}\text { Transporte público, mobilidade e planejamento urbano: contradições } \\
\text { essenciais } \\
\text { (Silveira y Cocco, 2013) }\end{array}$ & 2013 & 45 \\
\hline
\end{tabular}




\begin{tabular}{|c|c|c|}
\hline $\begin{array}{l}\text { The use of sustainability indicators in urban passenger transport during the } \\
\text { decision-making process: the case of Rio de Janeiro, Brazil } \\
\text { (Santos y Ribeiro, 2013) }\end{array}$ & 2013 & 44 \\
\hline $\begin{array}{l}\text { Economic, social and cultural transformation and the role of the bicycle in } \\
\text { Brazil } \\
\text { (Jones y Azevedo, 2013) }\end{array}$ & 2013 & 42 \\
\hline $\begin{array}{l}\text { The adoption of strategies for sustainable cities: a comparative study between } \\
\text { Newcastle and Florianópolis focused on urban mobility } \\
\text { (Guerra et al., 2016) }\end{array}$ & 2016 & 25 \\
\hline $\begin{array}{l}\text { A mobilidade urbana: uma agenda inconclusa ou o retorno daquilo que } \\
\text { não foi } \\
\text { (Gomide y Galindo, 2013) }\end{array}$ & 2013 & 24 \\
\hline $\begin{array}{l}\text { Mobilidade urbana: Um desafio paulistano } \\
\text { (Wilheim, 2013) }\end{array}$ & 2013 & 24 \\
\hline $\begin{array}{l}\text { Evaluation and Selection of Alternatives for the Promotion of Sustainable } \\
\text { Urban Mobility } \\
\text { (Lima, Lima y Silva, 2014) }\end{array}$ & 2014 & 21 \\
\hline $\begin{array}{l}\text { Mobilidade urbana: os desafios do futuro } \\
\text { (Silva, 2013) }\end{array}$ & 2013 & 21 \\
\hline $\begin{array}{l}\text { Prioritizing bicycle paths in Belo Horizonte City, Brazil: Analysis based on user } \\
\text { preferences and willingness considering individual heterogeneity } \\
\text { (Zhang, Magalhães y Wang, 2014) }\end{array}$ & 2014 & 20 \\
\hline $\begin{array}{l}\text { Avaliação multicritério da acessibilidade de pessoas com mobilidade } \\
\text { reduzida: um estudo na região central de Itajubá (MG) } \\
\text { (Machado y Lima, 2015) }\end{array}$ & 2015 & 19 \\
\hline $\begin{array}{l}\text { A Questão da Mobilidade Urbana nas Metrópoles Brasileiras } \\
\text { (Pero y Stefanelli, 2015) }\end{array}$ & 2015 & 18 \\
\hline $\begin{array}{l}\text { Mobilidade, Acessibilidade E Produtividade: Nota Sobre A Valoração } \\
\text { Econômica Do Tempo De Viagem Na Região Metropolitana De São Paulo } \\
\text { (Haddad y Vieira, 2015) }\end{array}$ & 2015 & 17 \\
\hline $\begin{array}{l}\text { Evaluating Restrictions on the Circulation of Freight Vehicles in Brazilian Cities } \\
\text { (Bontempo et al., 2014) }\end{array}$ & 2014 & 14 \\
\hline $\begin{array}{l}\text { Planos de mobilidade urbana: instrumento efetivo da política pública de } \\
\text { mobilidade? } \\
\text { (Lima Neto y Galindo, 2013) }\end{array}$ & 2013 & 13 \\
\hline $\begin{array}{l}\text { Um procedimento baseado na acessibilidade para a concepção de Planos } \\
\text { Estratégicos de Mobilidade Urbana: o caso do Brasil } \\
\text { (Mello y Portugal, 2017) }\end{array}$ & 2017 & 13 \\
\hline $\begin{array}{l}\text { A origem do caos? a crise de mobilidade no Rio de Janeiro e a ameaça à } \\
\text { saúde urbana } \\
\text { (Costa, Silva y Cohen, 2013) }\end{array}$ & 2013 & 12 \\
\hline $\begin{array}{l}\text { O padrão de mobilidade de São Paulo e o pressuposto de desigualdade } \\
\text { (Zandonade y Moretti, 2012) }\end{array}$ & 2012 & 12 \\
\hline $\begin{array}{l}\text { The consideration of environmental and social issues in transport policy, plan } \\
\text { and programme making in Brazil: A systems analysis } \\
\text { (Malvestio, Fischer y Montaño, 2018) }\end{array}$ & 2018 & 12 \\
\hline $\begin{array}{l}\text { Policy to promote bicycle use or bicycle to promote politicians? Bicycles in the } \\
\text { imagery of urban mobility in Brazil } \\
\text { (Medeiros y Duarte, 2013) }\end{array}$ & 2013 & 10 \\
\hline $\begin{array}{l}\text { Em busca do tempo perdido: Uma estimativa do produto perdido em } \\
\text { trânsito no Brasil } \\
\text { (Vianna y Young, 2015) }\end{array}$ & 2015 & 8 \\
\hline $\begin{array}{l}\text { Espaço, iniquidade e transporte público: avaliação da acessibilidade } \\
\text { urbana na cidade de Natal/RN por meio de indicadores de } \\
\text { sustentabilidade } \\
\text { (Costa y Morais, 2014) }\end{array}$ & 2014 & 7 \\
\hline
\end{tabular}




\begin{tabular}{|l|c|c|}
\hline $\begin{array}{l}\text { Centralidades urbanas e sistemas de transporte público em Goiânia, } \\
\text { Goiás } \\
\text { (Kneib, 2016) }\end{array}$ & 2016 & 6 \\
\hline $\begin{array}{l}\text { Mobilidade urbana e equidade social: possibilidades a partir das } \\
\text { recentes políticas de transporte público na Metrópole do Rio de Janeiro } \\
\text { (Silva, 2016) }\end{array}$ & 2016 & 5 \\
\hline $\begin{array}{l}\text { Analysis of Explanatory Variables of Rail Ridership: The Situation of Rio de } \\
\text { Janeiro } \\
\text { (Andrade, Gonçalves y Portugal, 2014) }\end{array}$ & 2014 & 4 \\
\hline $\begin{array}{l}\text { Motivos da escolha do automóvel no acesso a megaeventos: caso da } \\
\text { Copa das Confederações 2013, Rio de Janeiro } \\
\text { (Escobar, Flórez y Portugal, 2017) }\end{array}$ & 2017 & - \\
\hline $\begin{array}{l}\text { Estratégias para incentivar o transporte não motorizado em megaeventos } \\
\text { esportivos: o caso do estádio do Maracanã, Rio de Janeiro } \\
\text { (Flórez, Portugal y Escobar, 2018) }\end{array}$ & 2018 & - \\
\hline
\end{tabular}

Com o Portfólio Bibliográfico definido, inicia-se a segunda etapa, com a identificação de informações para gerar conhecimento quanto ao tema mobilidade urbana

\section{Etapa 2: Análise Bibliométrica}

O primeiro passo para análise bibliométrica é avaliar relevância dos periódicos: os quatro periódicos de destaque dos 17 presentes no Portfólio Bibliográfico são urbe: Revista Brasileira de Gestão Urbana, Estudos Avançados, Procedia - Social and Behavioral Sciences e Revista de Economia Contemporânea (Quadro 2), com cinco, quatro, três e três artigos publicados, respectivamente.

Quadro 2. Relevância dos periódicos presentes no Portfólio Bibliográfico (PB). Fonte: Elaboração própria.

\begin{tabular}{|l|c|}
\hline Periódicos & Quantidade de artigos do PB \\
\hline urbe. Revista Brasileira de Gestão Urbana & 5 \\
\hline Estudos Avançados & 3 \\
\hline Procedia - Social and Behavioral Sciences & 3 \\
\hline Revista de Economia Contemporânea & 4 \\
\hline
\end{tabular}

Dos periódicos presentes nas referências dos artigos do Portfólio Bibliográfico são destaque Transportation Research Part A: Policy and Practice, Journal of Transport Geography e o Procedia - Social and Behavioral Sciences (Quadro 3).

Quadro 3. Relevância dos periódicos das referências dos artigos do Portfólio Bibliográfico (PB). Fonte: Elaboração própria.

\begin{tabular}{|l|c|}
\hline Periódicos & Quantidade de artigos do PB \\
\hline Transportation Research Part A: Policy and Practice & 26 \\
\hline Journal of Transport Geography & 25 \\
\hline Procedia - Social and Behavioral Sciences & 8 \\
\hline
\end{tabular}

A partir das análises cruzadas realizadas, entre os periódicos do Portfólio Bibliográfico (PB) e dos periódicos das referências do PB pôde-se verificar que o periódico Procedia - Social and Behavioral Sciences, apresentou balanceado destaque dentre os demais periódicos, com três artigos do $\mathrm{PB}$ e 8 artigos das referências do $\mathrm{PB}$ na área de mobilidade urbana (Figura 4). 


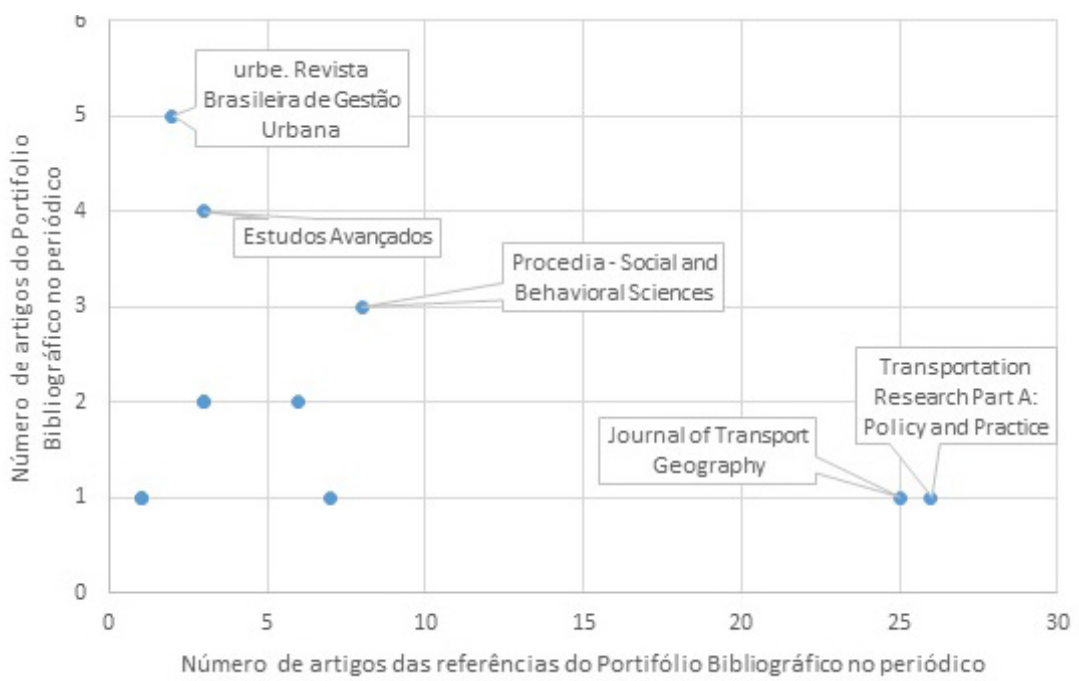

Figura 4. Comparação dos artigos do Portfólio Bibliográfico com os artigos das referências. Fonte: Elaboração Própria.

Ainda, uma análise considerada relevante pelos autores no processo de análise bibliométrica diz respeito ao fator de impacto dos periódicos na comunidade científica. Esse indicador informa o número médio de citações dos artigos publicados pelos periódicos ao longo de dois anos. No caso desta pesquisa, os periódicos que publicaram os artigos do Portfólio Bibliográfico foram consultados quanto aos seus fatores de impacto nas bases Web of Science e Scopus. Cada uma delas possui sua metodologia de cálculo e seu indicador próprio. Para a base Web of Science, o indicador utilizado é denominado Journal Citation Reports (JCR), e, na base Scopus, o indicador é o SCImago Journal Rank (SJR).

Para a base Web of Science, 12 dos 17 periódicos apresentam algum fator de impacto no meio acadêmico (Figura 5), como destaque o Transportation Research Part A: Policy and Practice e o Current Opinion in Environmental Sustainability.

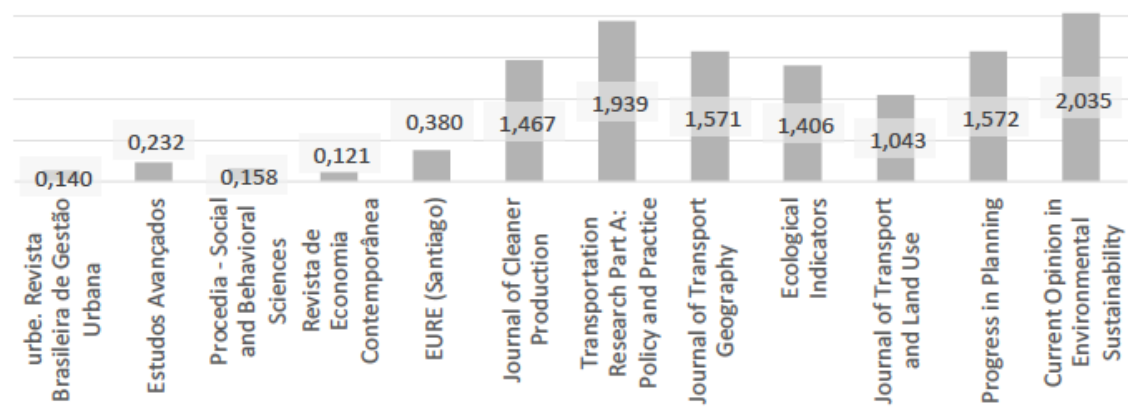

Figura 5. Fator de impacto Journal Citation Reports (JCR) dos periódicos do PB. Fonte: Elaboração Própria.

Já na base Scopus (Figura 6), 10 dos 17 periódicos do Portfólio Bibliográfico (PB) apresentaram algum fator de impacto em consulta realizada. Os periódicos com o maior fator de impacto entre os periódicos do PB são o Journal of Cleaner Production, Current Opinion in Environmental Sustainability e Ecological Indicators. 


\begin{tabular}{|c|c|c|c|c|c|c|c|c|c|}
\hline & & & \multicolumn{7}{|l|}{5,79} \\
\hline & & & & & & 4,42 & & & 4,87 \\
\hline & & & & 3,64 & 32, & & & 3,92 & \\
\hline & & & & & & & 2,05 & & \\
\hline 0,17 & 0,20 & 0,76 & & & & & & & \\
\hline & & . & & & & & & & \\
\hline 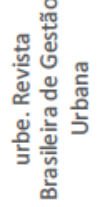 & 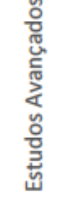 & 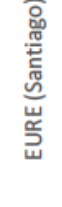 & 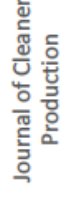 & 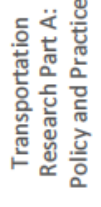 & 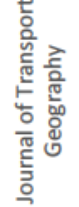 & 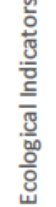 & 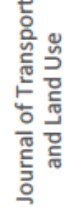 & 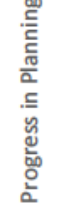 & 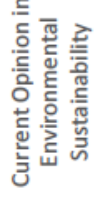 \\
\hline
\end{tabular}

Figura 6. Fator de impacto Scimago Journal Rank (SJR) dos periódicos do Portfólio Bibliográfico. Fonte: Elaboração Própria.

Ao se confrontarem as duas bases, apenas 10 dos 17 periódicos, possuem indicadores JCR e SJR, simultaneamente (Figura 7). Os periódicos com maior fator de impacto frente à comunidade científica em ambas as bases são o Transportation Research Part A: Policy and Practice, o Current Opinion in Environmental Sustainability, o Journal of Cleaner Production e o Ecological Indicators.

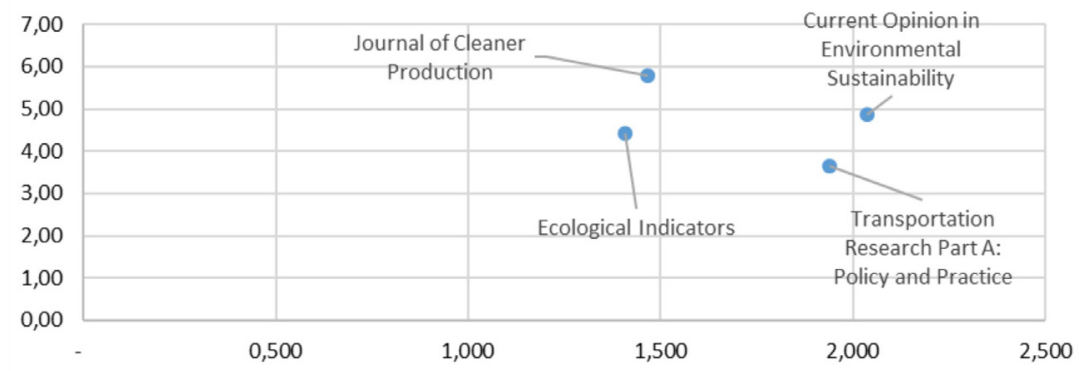

Figura 7. Fator de impacto cruzado JCR e Scopus dos periódicos do Portfólio Bibliográfico. Fonte: Elaboração Própria.

O próximo passo da análise se refere ao reconhecimento científico dos artigos do Portfólio Bibliográfico (PB) e sua identificação nas referências. No que tange aos artigos do PB, os trabalhos mais citados foram: The key to sustainable urban development in UK cities? The influence of density on social sustainability (Dempsey, Brown y Bramley, 2012), Linking urban transport and land use in developing countries (Cervero, 2013) e Urban sustainable transportation indicators for global comparison (Haghshenas y Vaziri, 2012) sendo que os três artigos destacados somados totalizam 53,6\% de todas as citações do PB (Quadro 4).

Quadro 4. Reconhecimento científico dos artigos do Portfólio Bibliográfico. Fonte: Elaboração própria.

\begin{tabular}{|l|l|}
\hline Artigos e autores & $\begin{array}{l}\% \text { do total } \\
\text { de citações } \\
\text { no PB }\end{array}$ \\
\hline $\begin{array}{l}\text { The key to sustainable urban development in UK cities? The influence of density on } \\
\text { social sustainability (Dempsey, Brown y Bramley, 2012) }\end{array}$ & $19,1 \%$ \\
\hline Linking urban transport and land use in developing countries (Cervero, 2013) & $19,0 \%$ \\
\hline $\begin{array}{l}\text { Urban sustainable transportation indicators for global comparison (Haghshenas y } \\
\text { Vaziri, 2012) }\end{array}$ & $15,5 \%$ \\
\hline
\end{tabular}


Em relação aos artigos das referências do Portfólio Bibliográfico (Quadro 5), constatouse que 28 , do total de 405 trabalhos, representam $50 \%$ de todas as citações indicadas pelo Google Acadêmico. Os artigos recentes de destaque são: Travel and the built environment; Encouraging pro-environmental behaviour: An integrative review and research agenda; Infrastructure, programs, and policies to increase bicycling: An international review; Current trends in smart city initiatives: Some stylised facts e The social dimension of sustainable development: Defining urban social sustainability, todos abordando dimensões transversais ao tema mobilidade urbana.

Quadro 5. Reconhecimento científico das referências do Portfólio Bibliográfico. Fonte: Elaboração Própria.

\begin{tabular}{|l|l|l|l|}
\hline Artigos e autores & Periódico & Ano & Citações \\
\hline $\begin{array}{l}\text { Travel And the Built Environment (Ewing y Cervero, } \\
\text { 2010) }\end{array}$ & $\begin{array}{l}\text { Journal of the American } \\
\text { Planning Association }\end{array}$ & 2010 & 2.574 \\
\hline $\begin{array}{l}\text { Encouraging pro-environmental behaviour: An } \\
\text { integrative review and research agenda (Steg y } \\
\text { Vlek, 2009) }\end{array}$ & $\begin{array}{l}\text { Journal of } \\
\text { Environmental } \\
\text { Psychology }\end{array}$ & 2009 & 2.509 \\
\hline $\begin{array}{l}\text { Infrastructure, programs, and policies to increase } \\
\text { bicycling: An international review (Pucher, Dill y } \\
\text { Handy, 2010)bike lanes and parking }\end{array}$ & Preventive Medicine & 2010 & 1.259 \\
\hline $\begin{array}{l}\text { Current trends in smart city initiatives: Some } \\
\text { stylised facts (Neirotti et al., 2014) }\end{array}$ & Cities & 2014 & 1.167 \\
\hline $\begin{array}{l}\text { The social dimension of sustainable development: } \\
\text { Defining urban social sustainability (Dempsey et } \\
\text { al., 2011) }\end{array}$ & $\begin{array}{l}\text { Sustainable } \\
\text { Development }\end{array}$ & 2011 & 1.063 \\
\hline
\end{tabular}

Quanto aos 63 autores do Portfólio Bibliográfico, destacaram-se Licínio da Silva Portugal, Josefina Flórez-Diaz, Josiane Palma Lima, Ernesto Pereira Galindo e Fábio Duarte, sendo que Licínio Portugal escreveu dois trabalhos em conjunto com Josefina Flórez-Diaz.

Quanto aos autores que figuram nas referências dos artigos, foram identificados 786 pesquisadores. Desses, destaca-se Robert Cervero, que figurou em 9 trabalhos das referências, sendo que um deles (Linking urban transport and land use in developing countries) também faz parte do Portfólio Bibliográfico.

Ao se cruzar os autores do Portfólio Bibliográfico (PB) com os autores que figuram na lista das referências do $\mathrm{PB}$, foi possível identificar dois autores de destaque tanto para o $\mathrm{PB}$, quanto para o referencial utilizado pelos artigos do PB, tais como Robert Cervero (com nove aparições nas referências e uma no portfólio) e Licínio Portugal (com seis trabalhos nas referências e um no $\mathrm{PB}$ ).

Quanto ao conteúdo dos 31 trabalhos do Portfólio Bibliográfico, foram identificados estudos com a preocupação com problemas urbanos como os crescentes congestionamentos, os acidentes de trânsito, a queda de produtividade, os problemas de saúde individual e coletiva, a degradação do meio ambiente, a periferização/ gentrificação nos grandes centros urbanos e a falta de cooperação/integração institucional. Em resposta aos desafios apresentados foram extraídos 22 grupos de medidas mitigadoras (Quadro 6). 
Quadro 6. Medidas mitigadoras identificadas no Portfólio Bibliográfico. Fonte: Elaboração Própria.

\begin{tabular}{|c|c|c|}
\hline \# & Grupo & Medidas mitigadoras \\
\hline 1 & A & Gestão política e governamental em mobilidade urbana \\
\hline 2 & B & Rezoneamento urbano em prol da mobilidade sustentável \\
\hline 3 & C & Reorganização de serviços / Transit Oriented Development \\
\hline 4 & D & Governança na mobilidade urbana \\
\hline 5 & $E$ & Priorização ao transporte não motorizado \\
\hline 6 & $\mathrm{~F}$ & Racionalização de veículos motorizados \\
\hline 7 & G & Faixas exclusivas de transporte coletivo \\
\hline 8 & $\mathrm{H}$ & Compartilhamento de veículos \\
\hline 9 & 1 & Políticas de intermodalidade \\
\hline 10 & $\mathrm{~J}$ & Restrição ao tráfego pesado \\
\hline 11 & K & Capacitação de motoristas e inspeção veicular \\
\hline 12 & $\mathrm{~L}$ & Participação de grupos sociais e horário de trabalho \\
\hline 13 & M & Impostos e taxas aos veículos motorizados \\
\hline 14 & $\mathrm{~N}$ & Gestão do conhecimento em mobilidade urbana \\
\hline 15 & $\mathrm{O}$ & Índices e indicadores de mobilidade urbana \\
\hline 16 & $\mathrm{P}$ & Gestão de tráfego \\
\hline 17 & Q & Avaliação ambiental estratégica em mobilidade \\
\hline 18 & $\mathrm{R}$ & Tecnologia veicular para sustentabilidade \\
\hline 19 & $\mathrm{~S}$ & Integração regional \\
\hline 20 & $\mathrm{~T}$ & Investimento em ampliação da oferta de transporte \\
\hline 21 & $U$ & Subsídios ao transporte \\
\hline 22 & V & Taxas e contribuições para arrecadação de recursos \\
\hline
\end{tabular}

A Política Nacional de Mobilidade Urbana, sancionada em 2012 com a Lei no 12.587 (Brasil, 2012), foi mencionada em 17 dos artigos, dentre sugestões e críticas quanto à implementação de suas diretrizes nos municípios brasileiros.

Quanto à metodologia, 23 dos artigos foram desenvolvidos como pesquisa bibliográfica/ documental descritiva e exploratória, 7 realizaram levantamento de dados em campo por meio de aplicação de formulários de pesquisa e um dos trabalhos foi de estudo de caso.

\section{Conclusões}

Com o intuito de atender os objetivos deste estudo foi necessário apresentar os periódicos, artigos e autores de maior destaque relacionados ao tema mobilidade urbana no período de 2012 a 2016. Dentre as limitações deste trabalho está: a coleta de dados até janeiro de 2019, sendo que a quantidade de citações pode ter sofrido alterações ao longo do ano; o uso do indexador "mobilidade urbana" em português na busca de artigos para composição do Portfólio Bibliográfico (contudo, foram selecionados 12 artigos em inglês).

Os resultados apresentados colaboram como ponto de partida para novas pesquisas relacionadas à mobilidade urbana. Os trabalhos citados servem como referencial teórico contemporâneo do tema, como também de temas transversais como o planejamento urbano e regional. Também foram mencionados os periódicos de maior impacto em mobilidade urbana, servindo como referência para submissão de novos trabalhos no tema. 
As dificuldades identificadas nos trabalhos convergem para problemas urbanos comuns como a gentrificação, o impacto na produtividade, na saúde e no meio ambiente. As oportunidades de pesquisas futuras surgem com a identificação da gama de medidas mitigadoras identificadas no Portfólio Bibliográfico, citadas no quadro 6, sendo esta a maior contribuição deste trabalho para a pesquisa acadêmica. 


\section{Q Bibliografía}

»Andrade, G. T. de, Gonçalves, J. A. M. y Portugal, L. Da S. (2014). Analysis of Explanatory Variables of Rail Ridership: The Situation of Rio de Janeiro. Procedia - Social and Behavioral Sciences, 162, 449-458.

»Araújo, C. A. (2006). Bibliometria : evolução histórica e questões atuais. Em Questão, 12 (1), 11-32.

"Barczak, R. y Duarte, F. (2012). Impactos ambientais da mobilidade urbana: cinco categorias de medidas mitigadoras. urbe. Revista Brasileira de Gestão Urbana, 4 (1), 13-32.

»Bontempo, A. P. et al. (2014). Evaluating Restrictions on the Circulation of Freight Vehicles in Brazilian Cities. Procedia - Social and Behavioral Sciences, 125, 275-283.

" Brasil. Ministério da Educação. Coordenação de Aperfeiçoamento de Pessoal de Nível Superior (Capes). Portal de Periódicos CAPES/MEC. Recuperado de https://www.periodicos.capes.gov.br/ (28/10/2019).

" Brasil. Lei no 12.587, de 3 de janeiro de 2012. Institui as diretrizes da Política Nacional de Mobilidade Urbana. Recuperado de http://www.planalto.gov.br/ ccivil_03/_ato2011-2014/2012/lei/l12587.htm (30/10/2019)

»Cervero, R. (2013). Linking urban transport and land use in developing countries. Journal of Transport and Land Use, 6 (1), 7-24.

»Costa, L. P. da y Morais, I. R. D. (2014). Espaço, iniquidade e transporte público: avaliação da acessibilidade urbana na cidade de Natal/RN por meio de indicadores de sustentabilidade. Sociedade $\&$ Natureza, 26 (2), 237-251.

" Costa, R. G.-R., Silva, C. G. T. da y Cohen, S. C. (2013). A origem do caos? a crise de mobilidade no Rio de Janeiro e a ameaça à saúde urbana. Cadernos Metrópole, 15 (30), 411-431.

"Dempsey, N. et al. (2011). The social dimension of sustainable development: Defining urban social sustainability. Sustainable Development, 19 (5), 289-300.

"Dempsey, N., Brown, C. y Bramley, G. (2012). The key to sustainable urban development in UK cities? The influence of density on social sustainability. Progress in Planning, 77 (3), 89-141.

»Ensslin, L., Ensslin, S. R. y Pinto, H. de M. (2013). Processo de investigação e análise bibliométrica: avaliação da qualidade dos serviços bancários. Revista de Administração Contemporânea, 17 (3), 325-349.

»Escobar, N., Flórez, J. y Portugal, L. da S. (2017). Motivos da escolha do automóvel no acesso a megaeventos: caso da Copa das Confederações 2013, Rio de Janeiro. urbe. Revista Brasileira de Gestão Urbana, 9 (1), 68-82.

» Ewing, R. y Cervero, R. (2010). Travel and the Built Environment. Journal of the American Planning Association, 76 (3), 265-294.

"Flórez, J., Portugal, L. da S., Escobar, N. (2018). Estratégias para incentivar o transporte não motorizado em megaeventos esportivos: o caso do estádio do Maracanã, Rio de Janeiro. urbe. Revista Brasileira de Gestão Urbana, 10 (2), 357-370. 
» Gomide, A. de Á. y Galindo, E. P. (2013). A mobilidade urbana: uma agenda inconclusa ou o retorno daquilo que não foi. Estudos Avançados, 27 (79), 27-39.

"Google. Google Acadêmico. Recuperado em https://scholar.google.com.br/ schhp?hl=pt-BR\&as_sdt=0,5 (28/10/2019).

" Guerra, J. B. S. O. de A. et al. (2016). The adoption of strategies for sustainable cities: a comparative study between Newcastle and Florianópolis focused on urban mobility. Journal of Cleaner Production, 113, 681-694.

» Haddad, E. A. y Vieira, R. S. (2015). Mobilidade, Acessibilidade e Produtividade: Nota sobre a Valoração Econômica do Tempo de Viagem na Região Metropolitana de São Paulo. Revista de Economia Contemporânea, 19 (3), 343-365.

» Haghshenas, H. y Vaziri, M. (2012). Urban sustainable transportation indicators for global comparison. Ecological Indicators, 15 (1), 115-121.

»Instituto Brasileiro de Geografia e Estatística (IBGE). Censo demográfico 2010. Recuperado em http://www.ibge.gov.br/home/estatistica/populacao/ censo2010/default.shtm (28/10/2019)

" Jones, T.y Azevedo, L. N. de. (2013). Economic, social and cultural transformation and the role of the bicycle in Brazil. Journal of Transport Geography, 30, 208-219.

»Kneib, E. C. (2016). Centralidades urbanas e sistemas de transporte público em Goiânia, Goiás. urbe. Revista Brasileira de Gestão Urbana, 8 (3), 306-317.

»Lima, J. P., Lima, R. Da S. y Silva, A. N. R. da. (2014). Evaluation and Selection of Alternatives for the Promotion of Sustainable Urban Mobility. Procedia - Social and Behavioral Sciences, 162, 408-418.

"Lima Neto, V. C. y Galindo, E. P. (2013). Planos de mobilidade urbana: instrumento efetivo da política pública de mobilidade? Paranoá: Cadernos de Arquitetura e Urbanismo, 9.

» Machado, M. H. y Lima, J. P. (2015). Avaliação multicritério da acessibilidade de pessoas com mobilidade reduzida: um estudo na região central de Itajubá (MG). urbe. Revista Brasileira de Gestão Urbana, 7 (3), 368-382.

" Malvestio, A. C., Fischer, T. B. y Montaño, M. (2018). The consideration of environmental and social issues in transport policy, plan and programme making in Brazil: A systems analysis. Journal of Cleaner Production, 179, 674-689.

» Marafon, A. D. et al. (2012). Revisão Sistêmica da Literatura sobre Avaliação de Desempenho na Gestão de P\&D. Revista Gestão Industrial, 8 (3).

» Medeiros, R. M. y Duarte, F. (2013). Policy to promote bicycle use or bicycle to promote politicians? Bicycles in the imagery of urban mobility in Brazil. Urban, Planning and Transport Research, 1 (1), 28-39.

" Mello, A. y Portugal, L. (2017). Um procedimento baseado na acessibilidade para a concepção de Planos Estratégicos de Mobilidade Urbana: o caso do Brasil. EURE (Santiago), 43 (128), 99-125.

» Neirotti, P. et al. (2014). Current trends in Smart City initiatives: Some stylised facts. Cities, 38, 25-36.

»Pero, V. y Stefanelli, V. A (2015). Questão da Mobilidade Urbana nas Metrópoles Brasileiras. Revista de Economia Contemporânea, 19 (3), 366-402.

"Pucher, J., Dill, J. y Handy, S. (2010). Infrastructure, programs, and policies to increase bicycling: An international review. Preventive Medicine, 50, S106-S125.

» Rubim, B. y Leitão, S. (2013). O plano de mobilidade urbana e o futuro das 
cidades. Estudos Avançados, 27 (79), 55-66.

"Santos, A. S. y Ribeiro, S. K. (2013). The use of sustainability indicators in urban passenger transport during the decision-making process: the case of Rio de Janeiro, Brazil. Current Opinion in Environmental Sustainability, 5 (2), 251-26o.

"Silva, A. da. (2016). Mobilidade urbana e equidade social: possibilidades a partir das recentes políticas de transporte público na Metrópole do Rio de Janeiro. GOT - Revista de Geografia e Ordenamento do Território, 10, 293-317.

»Silva, F. N. da. (2013). Mobilidade urbana: os desafios do futuro. Cadernos Metrópole, 15 (30), 377-388.

» Silveira, M. R. y Cocco, R. G. (2013). Transporte público, mobilidade e planejamento urbano: contradições essenciais. Estudos Avançados, 27 (79), $41-53$.

"Steg, L. y Vlek, C. (2009). Encouraging pro-environmental behaviour: An integrative review and research agenda. Journal of Environmental Psychology, 29 (3), 309-317.

"Vianna, G. S. B. y Young, C. E. F. (2015). Em Busca do Tempo Perdido: Uma Estimativa do Produto Perdido em Trânsito no Brasil. Revista de Economia Contemporânea, 19 (3), 403-416.

"Wilheim, J. (2013). Mobilidade urbana: um desafio paulistano. Estudos Avançados, 27 (79), 7-26.

»Zandonade, P. y Moretti, R. (2012). O padrão de mobilidade de São Paulo e o pressuposto de desigualdade. EURE (Santiago), 38 (113), 77-97.

» Zhang, D., Magalhães, D. J. A. V. y Wang, X. (Cara) (2014). Prioritizing bicycle paths in Belo Horizonte City, Brazil: Analysis based on user preferences and willingness considering individual heterogeneity. Transportation Research Part A: Policy and Practice, 67, 268-278.

\section{Victor Hugo-Pereira / vpereira@alunos.utfpr.edu.br}

Bacharel em Administração pelo Centro Universitário Franciscano do Paraná (FAE), Especialização em Gestão Pública pela Escola Nacional de Administração Pública (ENAP/Ministério do Planejamento) e Mestre em Planejamento e Governança Pública pela Universidade Tecnológica Federal do Paraná (UTFPR), Brasil. 